Crack tip fields in a material with three independent slip systems: NiAl single crystal

This content has been downloaded from IOPscience. Please scroll down to see the full text. 1992 Modelling Simul. Mater. Sci. Eng. 153

(http://iopscience.iop.org/0965-0393/1/1/006)

View the table of contents for this issue, or go to the journal homepage for more

Download details:

IP Address: 18.101.24.111

This content was downloaded on 01/07/2014 at 19:39

Please note that terms and conditions apply. 


\title{
Crack tip fields in a material with three independent slip systems: NiAl single crystal
}

\author{
Maryam Saeedvafał and James R Rice \\ Division of Applied Sciences, Harvard University, Cambridge, MA 02138, USA
}

Received 4 April 1992

\begin{abstract}
The crack tip stress and deformation field is analyzed for an ideally plastic ordered NiAl single crystal of B2 (BCC type) structure which has only three independent slip systems at room temperature, the $\{110\}(100)$ systems. For the crack on the $(010)$ plane growing in the [101] direction, only one of these systems is capable of sustaining considerable plane plastic flow. It involves slip planes lying parallel to the crack tip, making an angle of $90^{\circ}$ with the crack plane, and has the yield condition $\sigma_{12}= \pm \tau_{0}$ ( 1 is the cracking direction, 2 the crack plane normal, and $r_{0}$ the critical resolved shear stress). An elastic-ideally plastic asymptotic solution is derived in which the stress field has a ln $r$ type singularity, with a shearing discontinuity at $90^{\circ}$. These features are verified and more fully quantified by a finite element solution. At regions very near the tip, the field is divided into 5 angular sectors of which 2 are plastic regions $\left(\sigma_{12}= \pm \tau_{0}\right)$, one inclined ahead of the crack front and one behind. Further away from the tip, at distances greater than about 0.01 of the overall plastic zone size, the plastic region ahead of the crack front, in which $\sigma_{12}=\tau_{0}$, disappears resulting in 3 angular sectors of which one is plastic, which persists for the rest of the plastic region. While the plastic zone covers a broad angular range, the deformation field within it is dominated by the shear discontinuity of displacement.

The stress on other possible systems such as $\{110\}\langle 111\}$, which in NiAl becomes activated at higher temperatures, and on $\{112\}\langle 111\rangle$ systems, which are the usual twinning systems for BCC crystals, is also investigated. The results show that zones over which the resolved shear stresses on these two set of systems become of the order of critical resolved shear stress on the soft $\{110\}\langle 100\rangle$ system are either much smaller than, or of the same order as, the plastic zone for the $\{110\}\langle 100\rangle$ system, although very close to the crack tip the stresses on some of these systems become significant due to the stress singularity. Thus, even though the shear strength is much larger for these two systems than for the $\{110\}\langle 100\}$ system, over regions extremely near the tip they may become activated and alleviate the stress singularity. It is also possible that cleavage will occur first at lower temperatures or higher strain rates, and such is consistent with the brittleness of NiAl.
\end{abstract}

\section{Introduction}

Intermetallic ordered alloys have received much attention recently due to their high strength, high melting point, low density and environmental stability. Of these, B2 structured (BCC type) NiAl has limited room temperature ductility, which reduces its range of application. The $\{110\}\langle 100\rangle$ systems are the active slip systems for NiAl single crystals at room temperature as found by various researchers (Vedula 
and Stephens 1987, Vedula et al 1989, Guha et al 1989, Fu and Yoo 1991, Miracle et al 1989, George and Liu 1991, Russell et al 1989). These constitute only three independent systems, which are in general insufficient to fully alleviate the crack tip stress singularity, since a minimum of five systems are required to accommodate an arbitrary strain field. At higher temperatures (above $800 \mathrm{~K}$ at normal laboratory strain rates, of the order of $10^{-4} \mathrm{~s}^{-1}$ ) or with the addition of other solutes such as $\mathrm{Cr}$ and Mn (Miracle et al 1989), the $\{110\}\langle 111\rangle$ system also becomes active, but due to the high APB energy of dissociation of the $\langle 111\rangle$ superdislocation into partials (see Fu and Yoo 1991), very little activity is observed on this system at lower temperatures. Since the ideal cleavage strength of $\mathrm{NiAl}$ single crystals is not particularly low (about $29 \mathrm{GPa}$ on the (001) plane as calculated by Fu and Yoo (1991) using electron density functional methods), the usual brittleness of these crystals is generally thought to be the result of an insufficient number of slip systems at room temperature.

In this paper the crack tip field in NiAl single crystals, with the crack on the (001) cleavage plane, is studied in an attempt to investigate the crack tip deformation mechanisms resulting when there is an insufficient number of slip systems. The results could describe any solid which can flow plastically only, or primarily, in response to shear stress $\sigma_{12}$, where direction 1 is the direction of crack growth and direction 2 is perpendicular to the crack plane. A simple elastic-ideally plastic single crystal material model is adopted, and is implemented within 'small geometry change' assumptions by both asymptotic and finite element analysis to determine the incompletely relaxed crack tip stress and deformation field.

\section{Finite element formulation}

A 'User Material' subroutine was written by Huang (1991) for use in the finite element code ABAQUS (1989) to incorporate single crystal plasticity laws. The following is a summary of the constitutive laws used in the options within this subroutine that are appropriate to the present 'small strain' analysis.

Total strain rate can be written as the sum of the elastic and plastic strain rates

$$
\dot{\epsilon}_{i j}=\dot{\epsilon}_{i j}^{\mathrm{e}}+\dot{\epsilon}_{i j}^{\mathrm{p}}
$$

The plastic strain rate can be described as the tensorial sum of the slip rates on all systems

$$
\dot{\epsilon}_{i j}^{\mathrm{p}}=\sum_{\alpha} \mu_{i j}^{\alpha} \dot{\gamma}^{\alpha}
$$

where $\dot{\gamma}^{\alpha}$ is the slip rate on system $\alpha$ and $\mu_{i j}^{\alpha}$ is the Schmid factor. That is

$$
\mu_{i j}^{\alpha}=\frac{1}{2}\left(s_{i}^{\alpha} n_{j}^{\alpha}+s_{j}^{\alpha} n_{i}^{\alpha}\right)
$$

in which $s_{i}^{\alpha}$ is the slip direction and $n_{i}^{\alpha}$ is normal to slip plane $\alpha$. The resolved shear stress on system $\alpha$ is

$$
\tau^{\alpha}=\sigma_{i j} \mu_{i j}^{\alpha}
$$


The stress rate is related to the elastic strain rate by way of

$$
\dot{\sigma}_{i j}=L_{i j k l} \dot{\epsilon}_{k l}^{\mathrm{e}}=L_{i j k l}\left(\dot{\epsilon}_{k l}-\dot{\epsilon}_{k l}^{\mathrm{p}}\right)
$$

where $L_{i j k l}$ is the elastic moduli tensor, taken as isotropic in what follows.

A slightly rate-dependent formulation used by Peirce et al (1983) has been adopted to describe the relation between rate of slip $\dot{\gamma}^{\alpha}$ on a system and the resolved shear stress $\tau^{\alpha}$ on that system, namely

$$
\dot{\gamma}^{\alpha}=\dot{\gamma}_{0}^{\alpha} \operatorname{sgn}\left(\tau^{\alpha}\right)\left|\tau^{\alpha} / \tau_{0}^{\alpha}\right|^{m} .
$$

Here $\dot{\gamma}_{0}^{\alpha}$ and $\tau_{0}^{\alpha}$ are constants and $m \rightarrow \infty$ denotes the rate independent limit, in which $\tau_{0}^{\alpha}$ could be interpreted as the critical resolved shear stress on the slip system $\alpha(m=100$ has been used in this calculation). This rate-dependent formulation eliminates problems with uniqueness, especially in the ideally plastic case for crystals. An implicit time integration proposed by Kanchi et al (1978) has been utilized in order to increase the extremely small time steps that would be required by explicit time integration. In this scheme the increment in slip accumulated during the interval $\Delta t$ is estimated as

$$
\Delta \gamma^{\alpha}=\left[(1-\theta) \dot{\gamma}_{t}^{\alpha}+\theta \dot{\gamma}_{t+\Delta t}^{\alpha}\right] \Delta t
$$

and $\theta=0.7$ has been used since this value yielded the most accurate results (see Kanchi et al (1978)). Furthermore, $\dot{\gamma}_{t+\Delta t}^{\alpha}$ has been expanded in a one-term Taylor series to linearize the incremental equations namely

$$
\dot{\gamma}_{t+\Delta t}^{\alpha}=\dot{\gamma}_{t}^{\alpha}+\left[\frac{\partial \dot{\gamma}^{\alpha}}{\partial \tau^{\alpha}}\right]_{t} \Delta \tau^{\alpha} .
$$

The incremental constitutive relation, from which the tangent stiffness matrix is constructed, is derived by substituting equation (2) into (5), integrating over the time step, and using the results with equations (4), (7) and (8) to eliminate $\Delta \gamma^{\alpha}$, and has the form

$$
\Delta \sigma_{i j}=\hat{L}_{i j k l}\left[\Delta \epsilon_{k l}-\Delta t \sum_{\alpha} \mu_{k l}^{a} \dot{\gamma}_{t}^{\alpha}\right]
$$

where

$$
\hat{L}_{i j k l}=\left[L_{i j k l}^{-1}+\frac{\theta \Delta t}{m} \sum_{\alpha} \mu_{i j}^{\alpha} \mu_{k l}^{\alpha} \dot{\gamma}_{t}^{\alpha} / \tau_{t}^{\alpha}\right]^{-1} .
$$

Also, from equation (6), $\left[\partial \dot{\gamma}^{\alpha} / \partial \tau^{\alpha}\right]_{t}=\left[\dot{\gamma}^{\alpha} / m \tau^{\alpha}\right]_{t}$ has been used in (9). The total strain increment, $\Delta \epsilon_{k l}$, accumulated at an interval $\Delta t$ is related to the increment of nodal displacements, $\Delta u_{l}$, in the finite element formulation through a matrix which is the derivative of the element shape, or displacement interpolation, matrix.

The loading range is assumed to be restricted such that the size of the plastic zone is always much smaller than the region over which the conventional crack tip $r^{-1 / 2}$ elastic singularity dominates, so that small scale yielding conditions are established. 
The problem could then be modeled as a semi-infinite crack in an infinite body. Here, it is modeled as a crack along a ray of a circular region of radius much larger than the size of any plastic zone developed in the solution. Due to symmetry, only the upper half domain is considered, with points constrained against opening displacements over the plane ahead of the crack. A semi-circular mesh consisting of four noded isoparametric elements is used, where the inner nodes of the first ring of clements adjacent to the crack tip all occupy the same initial position at the tip. The mesh (shown in figure 1) is divided into three zones and at the circular borders between them the angular range of elements is doubled with respect to the inner adjacent zone. Except for the very inner rings which have equal dimensions in the radial direction, the elements have an aspect ratio of 1. The mesh has 6959 nodes and 6768 elements. Mode I tractions are imposed on the outer elements with the mode I stress intensity factor, $K_{\mathrm{I}}$, as the loading parameter. The stress intensity factor is increased at a uniform rate $\dot{K}_{\mathrm{l}}$. If $T$ is the total time for the stress intensity factor to increase to the value at which the solution is examined and $\tau_{0}$ is the yield stress entering the yield criterion $\sigma_{12}= \pm \tau_{0}$ to be discussed, then it is possible to see from dimensional analysis that if 'time' is denoted by $t / T$, 'stress' by $\sigma_{i j} / \tau_{0}$, 'strain' by $\epsilon_{i j} /\left(\tau_{0} / \mu\right)$ and 'displacements' by $\mu u_{i} / \tau_{0}$, then only three dimensionless parameters enter the formulation. These are the Poisson ratio $\nu$, taken to be 0.3 , the exponent $m$, taken to be 100 , and the combination $\dot{\gamma}_{0} T /\left(\tau_{0} / \mu\right)$, taken as 1 in this analysis. The ratio $\tau_{0} / \mu$ does not otherwise enter because of the 'small strain' framework. It would enter a 'finite strain' analysis as a fourth dimensionless material parameter. The choice for $\dot{\gamma}_{0} T /\left(\tau_{0} / \mu\right)$ assures that negligible plastic strain will occur unless $\tau$ is driven towards, and slightly above, $\tau_{0}$ (but only slightly; the large $m$ assures, for example, that a $\tau$ which is $1 \%$ above $\tau_{0}$ produces a strain rate of $3 \dot{\gamma}_{0}, 5 \%$ above produces $130 \dot{\gamma}_{0}$, and $10 \%$ produces $\left.14 \times 10^{3} \dot{\gamma}_{0}\right)$. To assure that the stresses, strains and displacements output by the program are $\sigma_{i j} / \tau_{0}, \epsilon_{i j} /\left(\tau_{0} / \mu\right)$ and $\mu u_{i} / \tau_{0}$, each of $\tau_{0}, \mu$ and the loading time within the program are specified as unity, this being allowed for $\mu$ only because a 'small strain' analysis is done. Lengths such as $\mu u_{i} / \tau_{0}$ and distance $r$ from the tip are later normalized by $\left(K_{\mathrm{I}} / \tau_{0}\right)^{2}$ in presenting the results, where $K_{\mathrm{I}}$ is the maximum stress intensity factor for this calculation. A nominal size scale for the plastic zone can be obtained by setting $r$ to the value at which $\sigma_{12}$ of the elastic singular field reaches $-\tau_{0}$ along a line in the 2 direction passing the crack tip, giving a nominal radius of $K_{I}^{2} / 16 \pi \tau_{0}^{2} \approx 0.02\left(K_{I} / \tau_{0}\right)^{2}$. In the mathematical small scale yielding problem for a half-infinite crack in a full space, this is the only length dimension. In the finite element model there are lengths set by element size and by overall grid dimensions. The ratio of the second to the first is large here ( $23 \times 10^{3}$ for the smallest elements at the crack tip); here, it is attempted to make the first irrelevant by growing the plastic region large compared to it, and the second by keeping the plastic zone small compared to it.

\section{Asymptotic solution}

The crystal orientation considered is shown in figure $2(a)$, with the crack on the $(010)$ plane with its tip along the [ $\overrightarrow{101}]$ direction so as to intersect a slip plane. Figure $2(b)$ shows the projection of the crystal in the (101) plane. Only the top half of the crystal is shown due to symmetry. Traces of the $\{110\}$ planes of the potentially active slip systems are shown in figure $2(b)$. Systems capable of large plastic flow under plane 

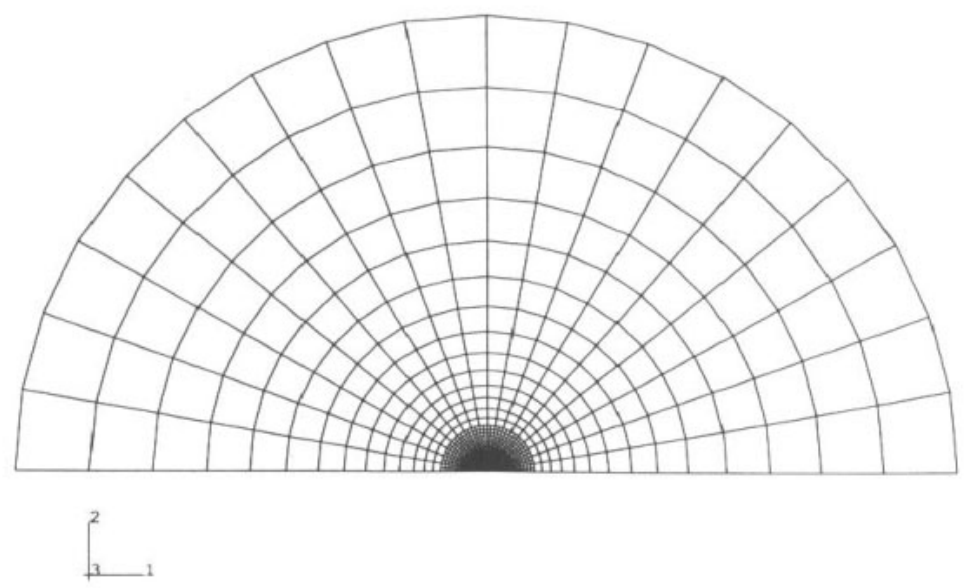

Figure 1. Finite element mesh,
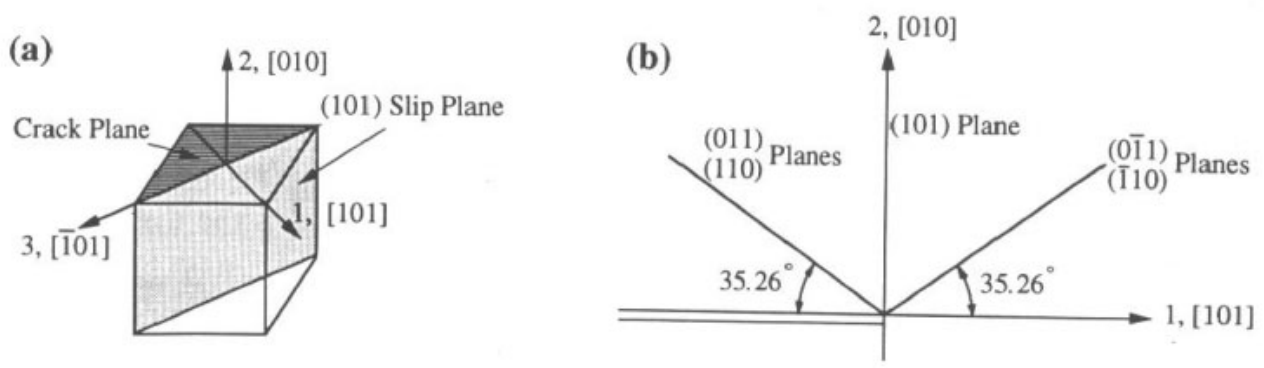

Figure 2. (a) Crystal orientation. (b) Projection of the crack configuration on the (i01) plane. Traces of the $\{110\}$ slip planes are marked.

strain conditions must have $\mu_{33}=0$. The slip system (101)[010] is the only one which does (except for the (101)[010] system which has $\mu_{11}=\mu_{22}=\mu_{12}=\mu_{33}=0$ and hence no shear stress resolved onto it by tensile loading). The yield surface, which is normally constructed by the inner envelope of all the lines of critical shear (resolved shear stress on a system, $\tau^{\alpha}=\tau_{0}$ ), is an unbounded surface, $\sigma_{12}= \pm \tau_{0}$, since all the Schmid factors are equal to zero except for $\mu_{12}$ for this system. Thus the only way that the elastic singularity can be (partially) alleviated is by plastic shearing on the (101)[010] system. Noting the features of a solution to the case of discontinuous shearing on an elastic half plane, as well as by looking at the finite element results discussed later, the crack tip field was assumed to have a logarithmic singularity. The field was assumed to be divided into angular sectors alternating between plastic and elastic regions.

In any plastic regions the shear stress $\sigma_{12}= \pm \tau_{0}$, thus the equilibrium equations $\left(\sigma_{i j}, j=0\right.$, where the third subscript, $j$, indicates the term is a partial derivative with respect to $x_{j}$, i.e. ()$\left.,_{j}=\partial() / \partial x_{j}\right)$ result in $\sigma_{11}=\sigma_{11}\left(x_{2}\right)$ and $\sigma_{22}=\sigma_{22}\left(x_{1}\right)$. Assuming that the field has a logarithmic singularity then

$$
\sigma_{11}=Z_{1} \ln \left|x_{2}\right|+Y_{1} \quad \sigma_{22}=Z_{2} \ln \left|x_{1}\right|+Y_{2}
$$


where $Z_{1}, Z_{2}, Y_{1}$ and $Y_{2}$ are constants. Now since the normal components of the plastic strain, $\epsilon_{11}^{\mathrm{p}}=\epsilon_{22}^{\mathrm{p}}=\epsilon_{33}^{\mathrm{p}}=0$, these strain components are entircly elastic and thus for plain strain as assumed here $\left(\epsilon_{33}=0\right)$

$$
\begin{aligned}
& \epsilon_{11}=u_{1,1}=\left[(1-\nu) \sigma_{11}-\nu \sigma_{22}\right] / 2 \mu \\
& \epsilon_{22}=u_{2,2}=\left[(1-\nu) \sigma_{22}-\nu \sigma_{11}\right] / 2 \mu \\
& \epsilon_{12}=\left(u_{1,2}+u_{2,1}\right) / 2=\sigma_{12} / 2 \mu+\epsilon_{12}^{\mathrm{p}}
\end{aligned}
$$

where $\nu$ is the Poisson ratio and $\mu$ is the shear modulus. Integrating the first two of the above equations leads to

$$
\begin{aligned}
& 2 \mu u_{1}=(1-\nu) \sigma_{11} x_{1}-\nu \int \sigma_{22}\left(x_{1}\right) \mathrm{d} x_{1}+f\left(x_{2}\right) \\
& 2 \mu u_{2}=(1-\nu) \sigma_{22} x_{2}-\nu \int \sigma_{11}\left(x_{2}\right) \mathrm{d} x_{2}+g\left(x_{1}\right) .
\end{aligned}
$$

Again since it is assumed that the field has a logarithmic singularity, $f$ and $g$ in the above equation are assumed to have the form

$$
f^{\prime}\left(x_{2}\right)=V_{1} \ln \left|x_{2}\right|+U_{1} \quad g^{\prime}\left(x_{1}\right)=V_{2} \ln \left|x_{1}\right|+U_{2}
$$

where a prime indicates the derivative of the function with respect to its argument and $V_{1}, V_{2}, U_{1}$ and $U_{2}$ are constants. Thus the full equations for the displacements are

$$
\begin{aligned}
2 \mu u_{1}= & {\left[(1-\nu) Z_{1} x_{1}+V_{1} x_{2}\right] \ln \left|x_{2}\right|-\nu Z_{2} x_{1} \ln \left|x_{1}\right| } \\
& +x_{1}\left[(1-\nu) Y_{1}-\nu Y_{2}+\nu Z_{2}\right]+x_{2}\left(U_{1}-V_{1}\right)+W_{1} \\
2 \mu u_{2}= & {\left[(1-\nu) Z_{2} x_{2}+V_{2} x_{1}\right] \ln \left|x_{1}\right|-\nu Z_{1} x_{2} \ln \left|x_{2}\right| } \\
& +x_{2}\left[(1-\nu) Y_{2}-\nu Y_{1}+\nu Z_{1}\right]+x_{1}\left(U_{2}-V_{2}\right)+W_{2}
\end{aligned}
$$

where $W_{1}$ and $W_{2}$ are integration constants. By differentiating the above equations the shear strain is obtained as

$$
\begin{aligned}
4 \mu \epsilon_{12}= \pm 2 \tau_{0} & +4 \mu \epsilon_{12}^{\mathrm{p}}=V_{1} \ln \left|x_{2}\right|+V_{2} \ln \left|x_{1}\right| \\
& +(1-\nu)\left[Z_{1}\left(x_{1} / x_{2}\right)+Z_{2}\left(x_{2} / x_{1}\right)\right]+U_{1}+U_{2} .
\end{aligned}
$$

The solution in the elastic regions is derived by using Muskhelishvili complex potentials which are

$$
\begin{aligned}
& \left(\sigma_{11}+\sigma_{22}\right) / 2=\operatorname{Re}\left[2 \phi^{\prime}(z)\right] \\
& \left(\sigma_{22}-\sigma_{11}\right) / 2+i \sigma_{12}=(\bar{z}-z) \phi^{\prime \prime}(z)-\phi^{\prime}(z)+\Omega^{\prime}(z) \\
& 2 \mu\left(u_{1}+i u_{2}\right)=(3-4 \nu) \phi(z)+(\bar{z}-z) \overline{\phi(z)^{\prime}}-\overline{\Omega(z)}
\end{aligned}
$$

where $z=x_{1}+\mathrm{i} x_{2}$ and the overbar denotes the complex conjugate of the function, and $\mathrm{i}=\sqrt{-1}$. Again due to the logarithmic singularity, it is assumed that $\phi^{\prime}=A \ln z+B$ and $\Omega^{\prime}=C \ln z+D$, where $A, B, C$ and $D$ are complex 
constants (e.g., $A=A_{1}+\mathrm{i} A_{2}$ ). The full equation of the field derived by using this assumed form of the complex potentials is

$$
\begin{aligned}
& 2 \mu u_{1}=[(3-\left.4 \nu) A_{1}-C_{1}\right] \cos \theta r \ln r-\left[(5-4 \nu) A_{2}-C_{2}\right] \sin \theta r \ln r \\
&+\left[C_{1}-(5-4 \nu) A_{1}\right] r \theta \sin \theta+\left[C_{2}-(3-4 \nu) A_{2}\right] r \theta \cos \theta \\
&+\left[(3-4 \nu)\left(B_{1}-A_{1}\right)+C_{1}-D_{1}\right] r \cos \theta \\
&-\left[(5-4 \nu) B_{2}-(3-4 \nu) A_{2}-D_{2}+C_{2}\right] r \sin \theta+E_{1} \\
& 2 \mu u_{2}=[(1-\left.4 \nu) A_{1}+C_{1}\right] \sin \theta r \ln r+\left[(3-4 \nu) A_{2}+C_{2}\right] \cos \theta r \ln r \\
&+\left[(3-4 \nu) A_{1}+C_{1}\right] r \theta \cos \theta-\left[(1-4 \nu) A_{2}+C_{2}\right] r \theta \sin \theta \\
&+\left[(1-4 \nu) B_{1}-(3-4 \nu) A_{1}+D_{1}-C_{1}\right] r \sin \theta \\
&+\left[(3-4 \nu)\left(B_{2}-A_{2}\right)+D_{2}-C_{2}\right] r \cos \theta+E_{2} \\
& \sigma_{11}=\left(3 A_{1}-\right.\left.C_{1}\right) \ln r+\left(C_{2}-3 A_{2}\right) \theta+A_{1}(1-\cos 2 \theta)-A_{2} \sin 2 \theta+3 B_{1}-D_{1} \\
& \sigma_{22}=\left(A_{1}+C_{1}\right) \ln r-\left(C_{2}+A_{2}\right) \theta-A_{1}(1-\cos 2 \theta)+A_{2} \sin 2 \theta+B_{1}+D_{1} \\
& \sigma_{12}=\left(C_{2}-A_{2}\right) \ln r+\left(C_{1}-A_{1}\right) \theta-A_{2}(1-\cos 2 \theta)-A_{1} \sin 2 \theta+D_{2}-B_{2}
\end{aligned}
$$

where $E_{1}$ and $E_{2}$ are integration constants and $z=r \exp (\mathrm{i} \theta)$.

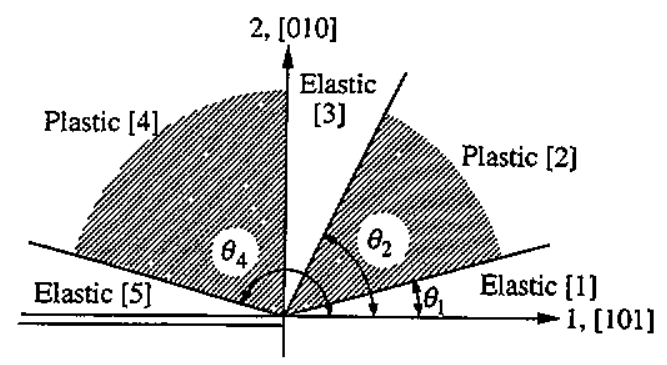

Figure 3. Sector arrangement in the asymptotic solution. The shaded regions are plastic sectors with region [2] at $\sigma_{12}=+\tau_{0}$ and region [4] at $\sigma_{12}=-\tau_{0}$.

The finite element results suggest that very near the crack tip the field is divided into five angular sectors as shown in figure 3 , where sectors [2] and [4] are plastic with $\sigma_{12}=+\tau_{0}$ in sector [2] and $\sigma_{12}=-\tau_{0}$ in sector [4]. Rice (1987) has proved that the only way a discontinuity can occur for ideally plastic single crystals is either along the direction of, or perpendicular to, the slip plane traces, and thus except for $\theta=90^{\circ}$ where a shear discontinuity can occur (across the slip system trace in the $x_{1-}$ $x_{2}$ plane), full continuity conditions must hold. That is, the stress and displacements are continuous at $\theta=\theta_{1}, \theta=\theta_{2}$ and $\theta=\theta_{4}$. At $\theta=90^{\circ}, \sigma_{11}, \sigma_{12}$ and $u_{1}$ must be continuous but $u_{2}$ and $\sigma_{22}$ need not be. On the crack faces $\sigma_{22}=\sigma_{12}=0$ (free surface boundary condition), and ahead of the crack $(\theta=0) \sigma_{12}=u_{2}=0$ due to symmetry. The full field equations in each of the sectors, which are derived by applying these boundary conditions, are given in the appendix. In those expressions the field in every sector is expressed in terms of the set of nine constants $Z_{1}, Z_{2}, W_{1}$, $W_{2}, X, Y$, and $\theta_{1}, \theta_{2}, \theta_{4}$ which appear in these equations. These constants coincide with some of the constants of the same label, introduced earlier, in specific sections; 
$X$ and $Y$ redefine constants $A_{1}$ and $B_{1}$ from sector [1]. In addition, the continuity conditions also yield the following relationships between $Z_{1}, Z_{2}, \theta_{1}, \theta_{2}$ and $\theta_{4}$

$$
\begin{aligned}
& \frac{1}{2}\left(Z_{1}-Z_{2}\right) \theta_{1}+\frac{1}{4}\left(Z_{1}+Z_{2}\right) \sin 2 \theta_{1}=-\tau_{0} \\
& \frac{1}{2}\left(Z_{1}-Z_{2}\right)\left(\pi / 2-\theta_{2}\right)-\frac{1}{4}\left(Z_{1}+Z_{2}\right) \sin 2 \theta_{2}=2 \tau_{0} \\
& \frac{1}{2} Z_{1}\left(\pi-\theta_{4}\right)-\frac{1}{4} Z_{1} \sin 2 \theta_{4}=-\tau_{0} \\
& Z_{1}\left(\cot \theta_{2}-\cot \theta_{1}\right)+Z_{2}\left(\tan \theta_{2}-\tan \theta_{1}\right)=0 .
\end{aligned}
$$

As can be seen, the field is not fully determined in the asymptotic solution but five constants are left undetermined, namely $\theta_{4}, W_{1}, W_{2}, X$ and $Y$. These can be found by matching the solution with the finite element results.

\section{Results of the finite element analysis}

Figure 4 shows contours of constant $\sigma_{12}$ values. Contour levels vary from $-1.02 \tau_{0}$ to $-0.90 \tau_{0}$. The plastic zone size is estimated here by the outline of the contour $\sigma_{12}=-0.96 \tau_{0}$, which has a maximum radius of $R_{\mathrm{p}}=0.044\left(K_{\mathrm{I}} / \tau_{0}\right)^{2}$, which is clearly within the small scale yielding range, since the outer edge of the mesh is at $r=0.40\left(K_{\mathrm{I}} / \tau_{0}\right)^{2}$. The level $-0.96 \tau_{0}$ was chosen as an effective measure of yield stress in the visco-plastic formulation since the contours show a rapid drop in $\sigma_{12}$ over a very small increase in radius but comparatively little increase of $\sigma_{12}$ with decreasing radius. Figure 5 is the enlargement of the near-tip region of figure 4 . As can be secn, the field is divided into angular regions with the plastic sectors falling inside of the contours of $\sigma_{12}= \pm \tau_{0}$. Near the tip (at distances of order of $r=3.0 \times 10^{-4}\left(K_{\mathrm{I}} / \tau_{0}\right)^{2}$ from the tip) there are five sectors as distinguished by the two plastic sectors, in agreement with the asymptotic assumptions. The plastic sector marked [4] in figure 3 of the asymptotic analysis is found from the finite element results to be located from $90^{\circ} \leqslant \theta \leqslant \theta_{4}=172.5^{\circ}$. The plastic sector marked [2] in the asymptotic analysis, which is located (when $r=0^{+}$) from $\theta_{1}=7.6^{\circ} \leqslant \theta \leqslant \theta_{2}=79.3^{\circ}$, vanishes as $r$ increases to $r=3.0 \times 10^{-4}\left(K_{\mathrm{I}} / \tau_{0}\right)^{2}$ from the tip. Thus at larger distances from the tip the field has only three angular regions and the end angle of plastic sector [4] is found to change to $\theta_{4}=116^{\circ}$. The angle $\theta_{4}$ is measured from figure 5 , while the angles $\theta_{1}$ and $\theta_{2}$ are calculated from equations (19) to (22) of the asymptotic solution as described below, and are in good agreement with what could be measured from figure 5 .

From the asymptotic analysis (equations (A.1) through (A.5) of the appendix) the following expressions can be compared to the finite element results, partly as a check and partly to determine the constants which were left undetermined by the asymptotic analysis:

$$
\begin{aligned}
{\left[u_{1}\right]_{\theta=\pi}=} & -(1-\nu) \frac{Z_{1} r}{2 \mu} \ln \left|\frac{r \sin \theta_{4} \sin \theta_{2}}{X \sin \theta_{1}}\right|+(r / 4 \mu)(1-\nu) \\
& \quad \times\left[2 Z_{1}+Z_{1} \sin ^{2} \theta_{4}-\left(Z_{1}+Z_{2}\right)\left(\cos ^{2} \theta_{2}+\sin ^{2} \theta_{1}\right)\right]+W_{1} / 2 \mu \\
{\left[u_{2}\right]_{\theta=\pi}=} & (r / 2 \mu)(1-\nu)\left[Z_{1}\left(\cot \theta_{4}+\cot \theta_{2}-\cot \theta_{1}\right)+Z_{1} \theta_{4}\right. \\
& \left.\quad-\left(3 Z_{1}+Z_{2}\right) \pi / 2+\left(Z_{1}+Z_{2}\right)\left(\theta_{2}-\theta_{1}\right)\right]+W_{2} / 2 \mu \\
{\left[\sigma_{11}\right]_{\theta=0}=} & Z_{1} \ln (r / X) \\
{\left[\sigma_{22}\right]_{\theta=0}=} & Z_{2} \ln (r / Y)
\end{aligned}
$$



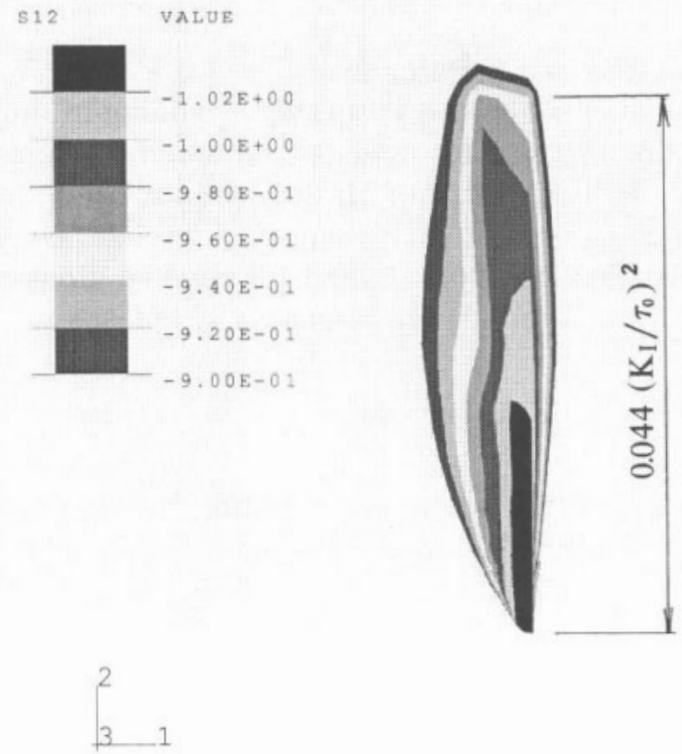

Figure 4. Contours of constant $\sigma_{12}$. Contour levels vary from $-1.02 \tau_{0}$ to $-0.90 \tau_{0}$.

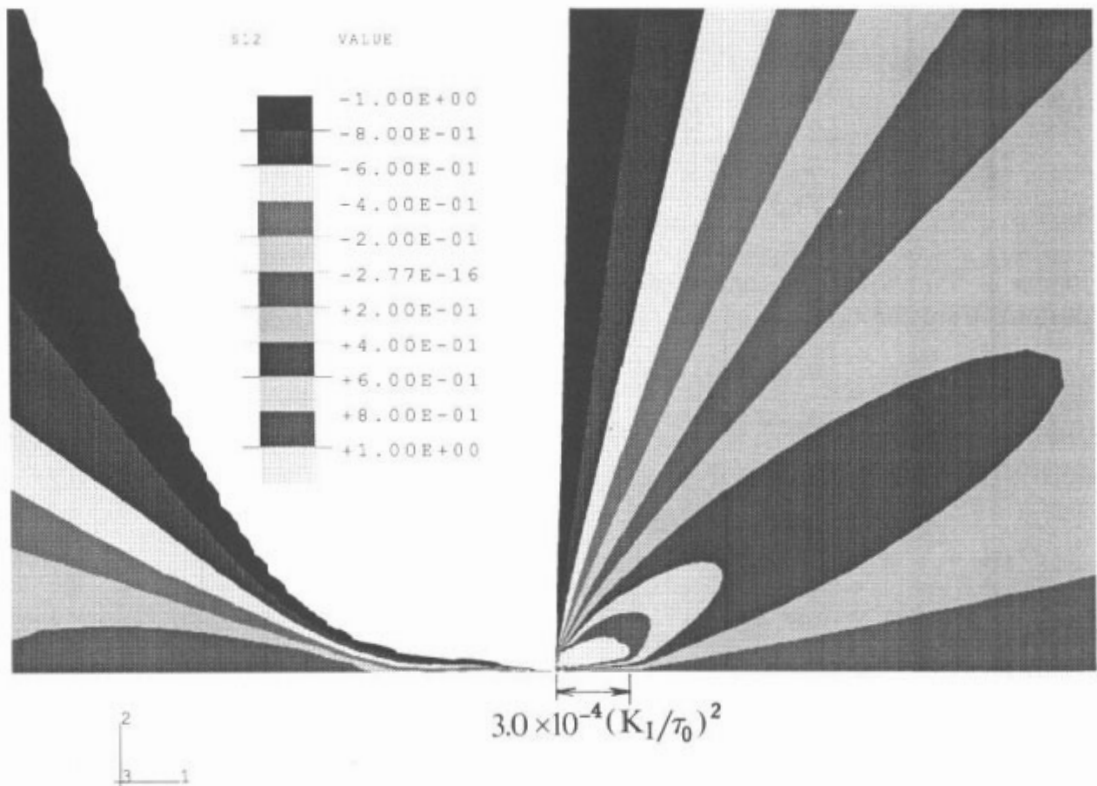

Figure 5. Enlargement of the near-tip area of contours of constant $\sigma_{12}$ near the crack tip. Contour levels vary from $-\tau_{0}$ to $\tau_{0}$.

for the case of five angular sectors. Independent of the considerations based on equations (23), substituting the value of $\theta_{4}=172.5^{\circ}$ as measured from figure 5 into equations (19) through (22) and simultaneously solving those equations results in 


$$
Z_{1}=-7.68 \tau_{0} \quad Z_{2}=-10.93 \tau_{0} \quad \theta_{1}=7.6^{\circ} \quad \theta_{2}=79.3^{\circ} .
$$

As described earlier, ar distances greater than $r=3.0 \times 10^{-4}\left(K_{\mathrm{I}} / \tau_{0}\right)^{2}$ from the crack tip the plastic zone ahead of the crack vanishes, resulting in only three angular sectors. The solution for this three-sector field can be obtained from the asymptotic analysis by letting $\theta_{1}=\theta_{2}$ (which will drop all dependence on $\theta_{1}$ or $\theta_{2}$ out of the equations). Then the field equations for plastic sector [2] become immaterial, and the resulting field equations for elastic sectors [1] and [3] become identical. Substituting $\theta_{4}=116.0^{\circ}$ (as measured from figure 5) into equation (21) leads to

$$
Z_{1}=-1.32 \tau_{0} \quad Z_{2}=-2.60 \tau_{0}
$$

for the case of three angular sectors, where the constant $Z_{2}$ was obtained by adding equations (19) and (20) to obtain $\left(Z_{1}-Z_{2}\right) \pi / 4=\tau_{0}$.

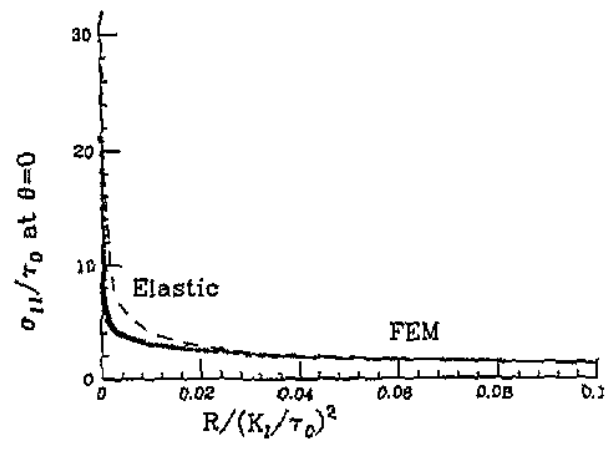

Figure 6. Variation of $\sigma_{11}$ ahead of the crack with distance from the tip.

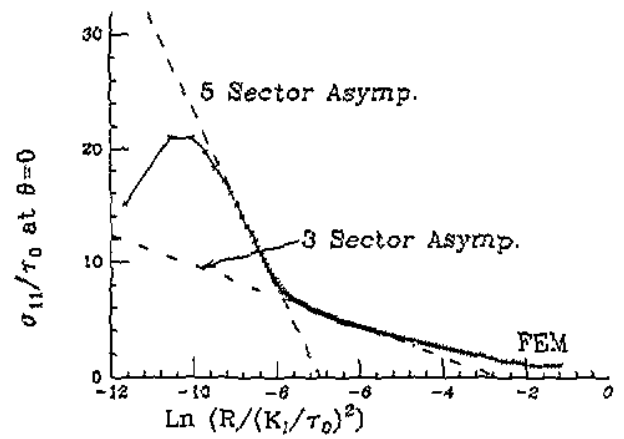

Figure 8. Variation of $\sigma_{11}$ ahead of the crack with distance from the tip on a logarithmic scale.

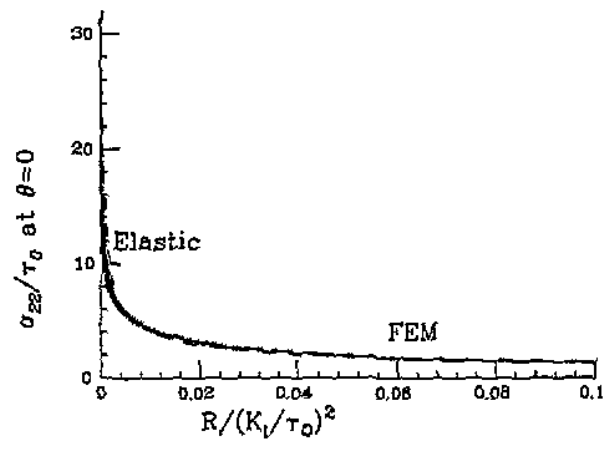

Figure 7. Variation of $\sigma_{22}$ ahead of the crack with distance from the tip.

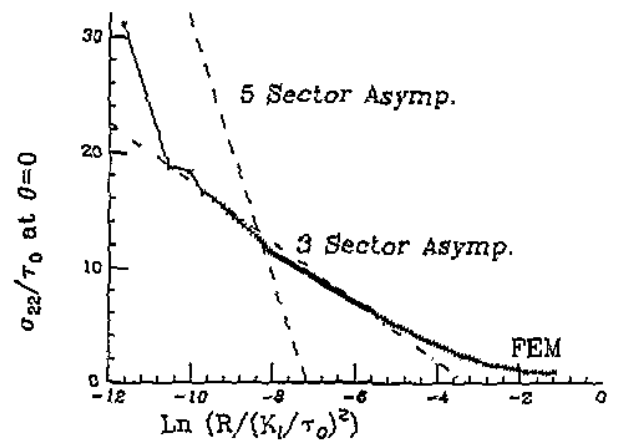

Eigure 9. Variation of $\sigma_{22}$ ahead of the crack with distance from the tip on a logaritimic scale. 
Figures 6 and 7 show the variation of normal stresses $\sigma_{11}$ and $\sigma_{22}$ ahead of the crack with distance from the tip. The dashed lines are the mode I elastic distribution of stresses ahead of the crack, $\sigma_{11}=\sigma_{22}=K_{1} / \sqrt{2 \pi r}$. Figures 8 and 9 are the same two figures on a logarithmic scale. The solid lines are the FEM results, the dashed lines are the results from the five angular sectors of the asymptotic solution, equations (23), and the dot-dashed lines are from the results of three angular sectors, based on $Z_{1}$ and $Z_{2}$ values given above. By positioning the dashed and dot-dashed lines to best coincide with the FEM results in different ranges, it is additionally determined that

$X=9.45 \times 10^{-4}\left(K_{1} / \tau_{0}\right)^{2} \quad Y=7.75 \times 10^{-4}\left(K_{1} / \tau_{0}\right)^{2}$

for the case of five angular sectors and

$$
X=0.0685\left(K_{1} / \tau_{0}\right)^{2} \quad Y=0.0371\left(K_{1} / \tau_{0}\right)^{2}
$$

for the case of three angular sectors.
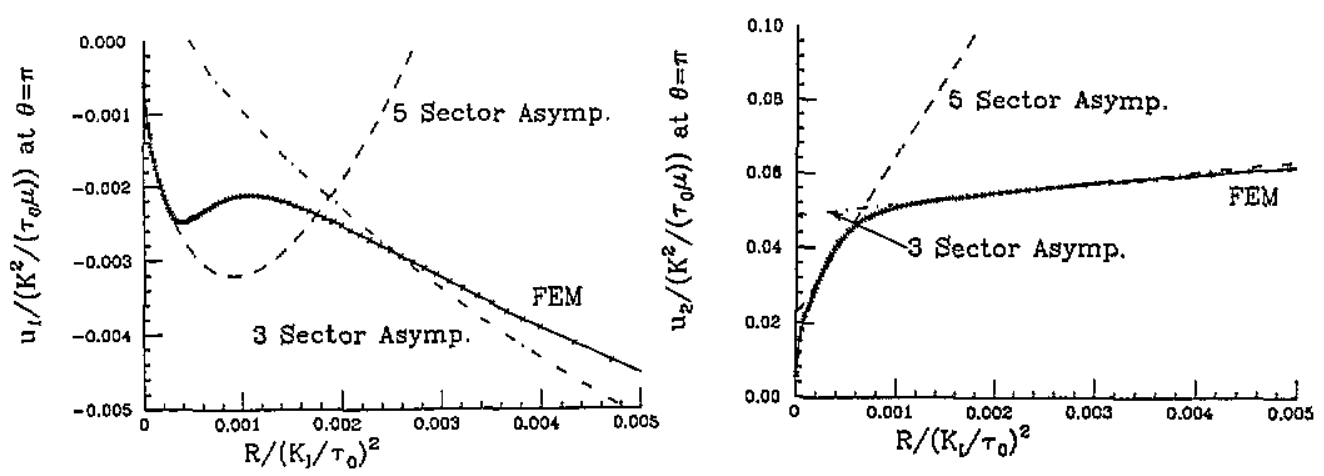

Figure 10. Variation of $u_{1}$ on the crack surface with distance from the tip.

Figure 11. Variation of $u_{2}$ on the crack surface with distance from the tip.

Figures 10 and 11 show the variations of the displacements $u_{1}$ and $u_{2}$ on the crack surface with distance from the tip. Again the solid lines are the FEM results, the dashed lines the results for five angular sectors and dot-dashed lines are the results for three angular sectors of the asymptotic solution. From these figures

$W_{1}=-1.48 \times 10^{-3}\left(K_{\mathrm{I}}\right)^{2} / \tau_{0} \quad \delta_{\text {tip }}=W_{2} / \mu=4.44 \times 10^{-2}\left(K_{1}\right)^{2} / \mu \tau_{0}$

for the case of five angular sectors, where $\delta_{\text {tip }}$ is the crack opening displacement $\left(\delta_{\text {tip }}=\left[2 u_{2}\right]_{\theta=\pi}\right.$ as $\left.r \rightarrow 0\right)$, and

$W_{1}=2.00 \times 10^{-3}\left(K_{\mathrm{I}}\right)^{2} / \tau_{0} \quad \delta_{\text {tip }}=W_{2} / \mu=9.72 \times 10^{-2}\left(K_{\mathrm{I}}\right)^{2} / \mu \tau_{0}$

for the case of three angular sectors.

As obvious from these figures, specially figures 5,8 and 11 , the field is clearly divided into two zones with different slopes in each as caused by the existence of the plastic sector [2] of small radial extent. For both the 3 and 5 angular sectors 
asymptotic solutions the results are in good agreement with the the finite element results, except for the variation of $\sigma_{22}$ where the solution is only an approximate match for the 5 angular sectors case (see figure 9). Note that the matching of the FEM and the asymptotic results for determining the constants $X, Y, W_{1}$ and $W_{2}$ from figures $8,9,10$ and 11 respectively, were done at one point for each zone (i.e. 5 or 3 angular sector zones) which fell well into its applicable range. For the case of the 5 angular sector zone, that meant a point which was not too close to the tip (since the FEM results at the elements very near the tip are not dependable), but at a distance smaller than $3.0 \times 10^{-4}\left(K_{\mathrm{I}} / \tau_{0}\right)^{2}$ (the end of plastic sector [2]). The point chosen was at a radius of $2.5 \times 10^{-4}\left(K_{\mathrm{I}} / \tau_{0}\right)^{2}$ which gave the best match of slopes for $\sigma_{11}$ and $u_{2}$. Although figure 9 shows that the first two points of the FEM results do match the predicted asymptotic slopes of $\sigma_{22}$, that slope comes from the first element at the crack tip, the result of which is unreliable (as can be seen best in figure 8).

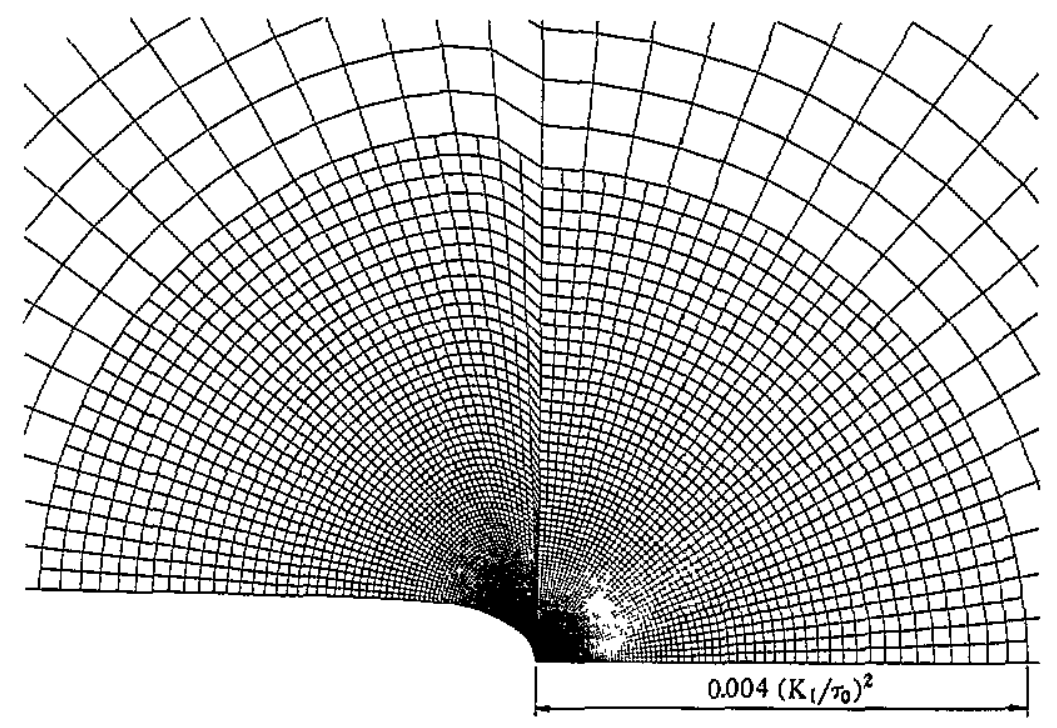

Figure 12. Displaced mesh near the crack tip.

The small plastic zone ahead of the tip in the 5 angular sector range, has a radial extent $R_{\mathrm{P}}^{\prime}=0.0068 R_{\mathrm{P}}=6.7 \times 10^{-3}\left(\mu / \tau_{0}\right) \delta_{\text {tip }}$. The 5-sector field would be a valid prediction of 'small strain' theory only at distances $r$ within it that are at least, say, three or more times $\delta_{\text {tip }}$, so as to be in the range not affected by crack tip blunting and associated large geometry changes. Such a range can exist at all for the 5-sector field only if $R_{\mathrm{P}}^{\prime}$ is several times larger than $\delta_{\text {tip }}$, which means $6.7 \times 10^{-3}\left(\mu / \tau_{0}\right)$ must be several times larger than unity. Since the polycrystalline tensile $0.2 \%$ yield strength for $\mathrm{NiAl}$ is around $200 \mathrm{MPa}$ at $300 \mathrm{~K}$, and around $100 \mathrm{MPa}$ at $300 \mathrm{~K}$ (Nocbe et al 1992), $\tau_{0}$ at those temperatures is estimated to be about $100 \mathrm{MPa}$ and $50 \mathrm{MPa}$, respectively. Also given a bulk modulus $B$ as $1.9 \times 10^{5} \mathrm{MPa}$ (Fleisher et al 1989), the shear modulus may be estimated as $\mu=9 \times 10^{4} \mathrm{MPa}$, assuming $\nu=0.3$. Thus $R_{\mathrm{P}}^{\prime} / \delta_{\text {tip }}=6.7 \times 10^{-3}\left(\mu / \tau_{0}\right)=6$ at $300 \mathrm{~K}$ and 12 at $600 \mathrm{~K}$, which provides at least some limited range of the 5-sector field which can presumably be described by the present 'small strain' analysis. 

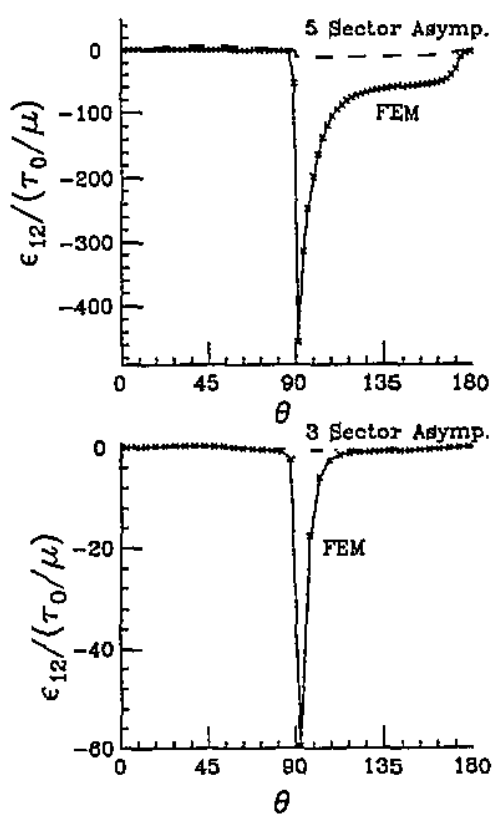

Figure 13. Variation of shear strain $\epsilon_{12}$ around the crack tip, for 5 and 3 angular sector zones.

Figure 12 shows the displaced mesh near the tip. Figure 13 shows the variation of $\epsilon_{12}$ around the crack tip at distances $r=2.5 \times 10^{-4}\left(K_{\mathrm{l}} / \tau_{0}\right)^{2}$ and $r=2.5 \times 10^{-3}\left(K_{\mathrm{I}} / \tau_{0}\right)^{2}$ from the crack tip for the case of 5 and 3 angular sectors, respectively. These distances, which are within the applicable ranges of the 5 and 3 angular sector zones, were the points chosen for comparing the asymptotic results with the finite elements. The solid lines in figure 13 are the finite element results, the dashed lines are the 5-sector asymptotic results, and the dot-dashed lines are the 3 -sector asymptotic results. The asymptotic results have a Dirac 'delta' singularity in $\epsilon_{12}$ at $\theta=90^{\circ}$, associated with the shear displacement discontinuity predicted there. As can be seen from these figures, there is a clear shear discontinuity across $\theta=90^{\circ}$ parallel to slip line trace of the (101)[010] system. Again there is a good agreement between the finite element results and the 3-sector asymptotic analysis, but only an approximate match between the FEM results and 5-sector asymptotic analysis.

Figure 14 shows contours of resolved shear stress on (110)[111] slip system, assumed not to be activated in the present solution. Figure 15 shows contours of resolved shear stress on (121)[111] slip system. Among the members of the $\{110\}\langle 111\rangle$ and $\{112\}\langle 111\rangle$ systems capable of sustaining large plastic flow $\left(\mu_{33}=0\right.$ which means $\epsilon_{33}^{p}=0$ for non-zero in-plane $\epsilon_{i j}^{\mathrm{p}}$ ), these two were chosen since they exhibited the largest zone over which the resolved shear stress is larger than $\tau_{0}$. Since the Schmid factor for some of the systems are the same in this orientation under plane strain conditions, the resolved shear stress is equal for some of these systems, thus figure 14 also shows the resolved shear stress on (011)[11̄1] system. Both of these two systems form an angle of $35.26^{\circ}$ with the crack face. The contour levels shown in figures 14 and 15 vary from $-2 \tau_{0}$ to $-\tau_{0}$, with the outermost contour representing $\tau=-\tau_{0}$. As can be seen in these two figures the largest zones in which resolved shear stress exceeds $\tau_{0}$ are of the order of plastic zone size $R_{\mathrm{p}}=0.044\left(K_{\mathrm{l}} / \tau_{0}\right)^{2}$. Figure 16 shows the variation of resolved shear stress on systems (110)[111] or (011)[111] 


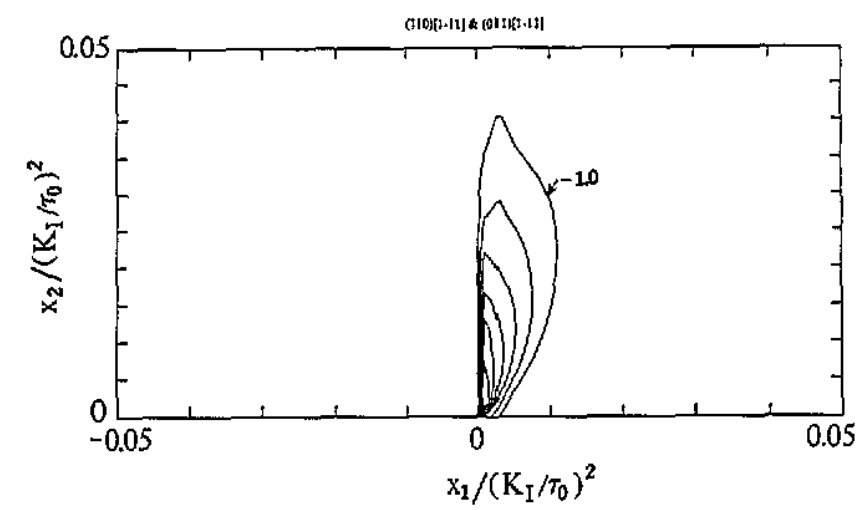

Figure 14. Contours of constant resolved shear stress on (110)[11̄1] or (011)[111] systems. Contour levels vary from $-2 \tau_{0}$ to $-\tau_{0}$.

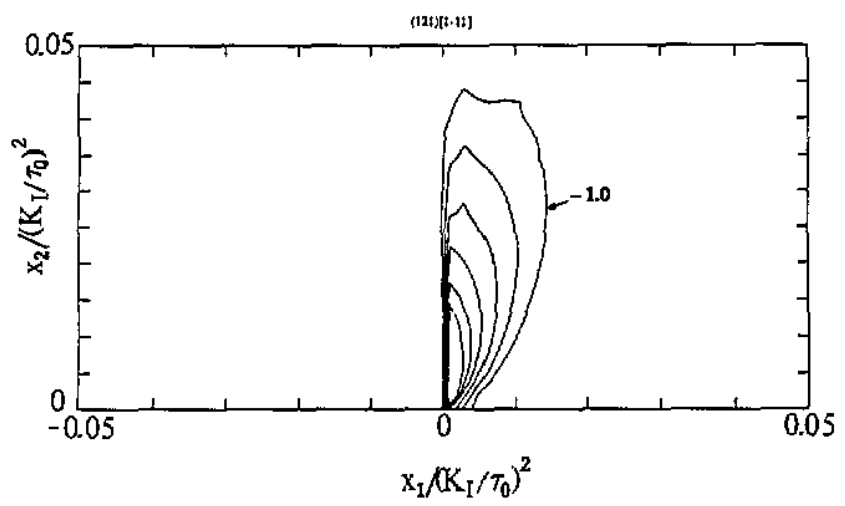

Figure 15. Contours of constant resolved shear stress on (121)[111] system. Contour levels vary from $-2 \tau_{0}$ to $-\tau_{0}$.

around the crack tip. Figure 17 shows the variation of resolved shear stress on system (121)[111] around the crack tip. The finite element results, shown as solid lines in figures 16 and 17 , are taken at distances $r=2.5 \times 10^{-4}\left(K_{\mathrm{I}} / \tau_{0}\right)^{2}$ and $r=2.5 \times 10^{-3}\left(K_{\mathrm{I}} / \tau_{0}\right)^{2}$ from the crack tip for the case of 5 and 3 angular sectors, respectively. The first distance is about $5.7 \delta_{\text {tip }}$ when $\tau_{0} / \mu=10^{-3}$. Again the dashed lines in these figures are the results for the 5 angular sectors of the asymptotic solution and the dot-dashed lines are the results for the 3 angular sector asymptotic solution. Figures 16 and 17 again show that the results for 5-sector angular asymptotic solution is only a qualitative match while there is a very good match between the finite element results and the 3 angular sector asymptotic results. Note that the Schmid factors for

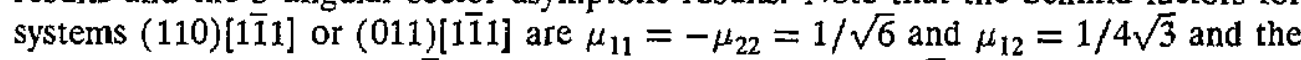
Schmid factors for (121)[111] systems are $\mu_{11}=-\mu_{22}=\sqrt{2} / 3$ and $\mu_{12}=1 / 6$. and thus the resolved shear stress on either of the two systems is

$$
\tau=A\left[\sigma_{11}-\sigma_{22}+(1 / \sqrt{2}) \sigma_{12}\right]
$$


where $A=1 / \sqrt{6}$ for (110)[1111] or (011)[1111] systems and $A=\sqrt{2} / 3$ for (121)[1 111$]$ system. Thus substituting the equations for $\sigma_{11}, \sigma_{22}$ and $\sigma_{12}$ in each of the sectors into equations (30) leads to

$$
\tau=A[f(\theta) \ln r+g(\theta)]
$$

where $f$ and $g$ are also dependent on the constants of each sector. Thus as can be seen from equation (31), as well as figures 16 and 17, the resolved shear stress in these two systems (as well as many other members of their respective set of systems) is infinite at the crack tip due to the singular stress field. Realistically, one should probably cutoff the $\ln r$ at a distance of $r \approx 2 \delta_{\text {tip }}$. Thus flow on these two systems could contribute to alleviate the unrelaxed crack tip stresses, even though the critical resolved shear stress on these systems is much larger than that of the $\{110\}\langle 100\rangle$ slip system at room temperature. Note that there are 7 independent slip systems in the $\{112\}\langle 111\rangle$ family and 5 independent slip systems in the $\{110\}\langle 111\rangle$ family with 4 and 3 systems in each respectively which are capable of sustaining large plastic flow. Thus activation of either of these two systems is sufficient for a bounded stress field. The tensile stress directly ahead of the crack is about $\sigma_{22}=15.5 \tau_{0}$, from finite element results of figure 9 , at the cut off distance $r=2 \delta_{\text {tip }}$, which for $\left(\mu / \tau_{0}\right)=10^{-3}$, corresponds to $\ln \left[r /\left(K_{\mathrm{I}} / \tau_{0}\right)^{2}\right]=-9.3$. The competition at the crack tip is between cleavage, aided by such large stresses, and plastic relaxation.

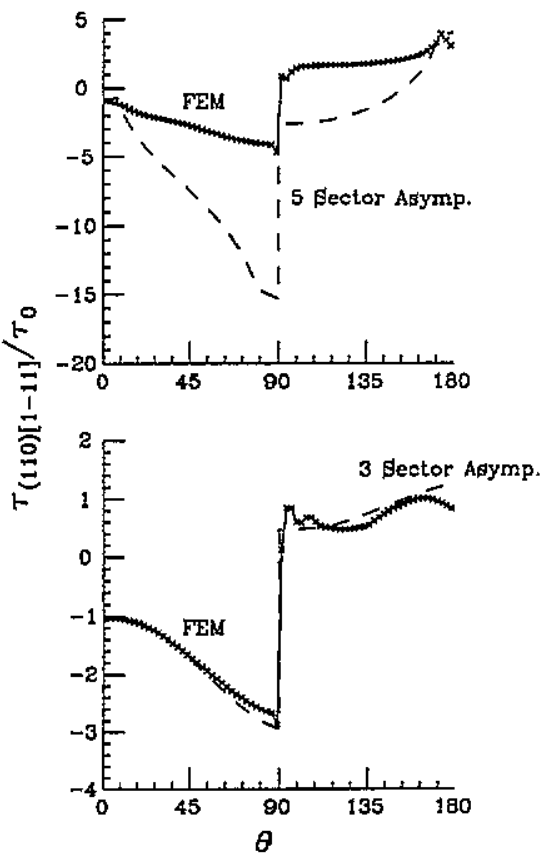

Figure 16. Variation of resolved shear stress on (110) $[1 \overline{1} 1]$ or $(011)[1 \overline{1} 1]$ systems around the crack tip, for 5 and 3 angular sector zones.

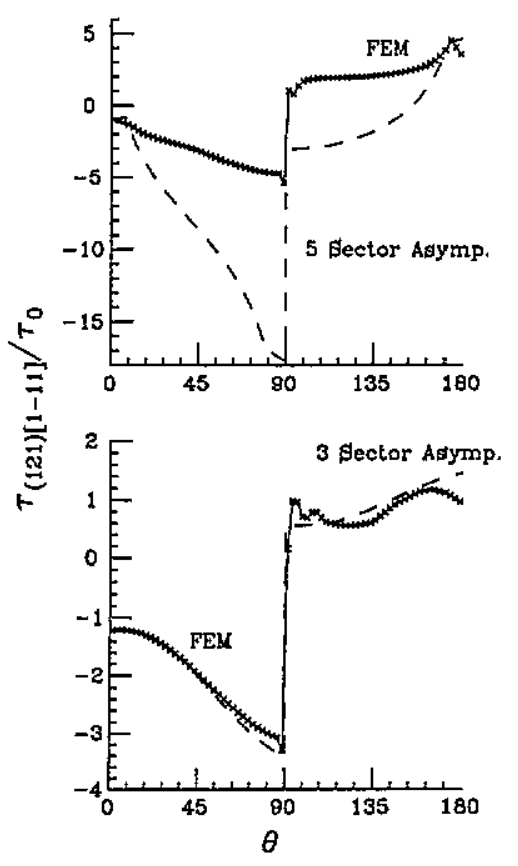

Figure 17. Variation of resolved shear stress on (121) $[1 \overline{1} 1]$ system around the crack tip, for 5 and 3 angular sector zones. 


\section{Conclusions}

The asymptotic as well as the finite element results show that the elastic-ideally plastic NiAI single crystal with the crack on the (010) plane growing in the [101] direction has a ln $r$ type singularity in the stresses, at least within the 'small strain' theory, when there is only one system, (101)[010], which is capable of sustaining large plastic flow. The field has a distinct shear displacement discontinuity at $\theta=90^{\circ}$ along the slip plane trace of the (101)[010] system and is divided into two zones, an inner one containing 5 angular sectors alternating from elastic to plastic, and an outer one with 3 angular sectors. The zone in which the solution contains 5 angular sectors has two plastic sectors, one at $7.6^{\circ} \leqslant \theta \leqslant 79.3^{\circ}$, vanishing at distances $R_{\mathrm{P}}^{\prime}=3.0 \times 10^{-4}\left(K_{\mathrm{I}} / \tau_{0}\right)^{2}$ from the tip, in which $\sigma_{12}=+\tau_{0}$, and one at $90^{\circ} \leqslant \theta \leqslant 172.5^{\circ}$ in which $\sigma_{12}=-\tau_{0}$. Over distances larger than $R_{\mathrm{p}}^{\prime}$ from the tip the field is divided into 3 angular sectors with the plastic sector at $90^{\circ} \leqslant \theta \leqslant 116^{\circ}$ in which $\sigma_{12}=-\tau_{0}$, vanishing at $R_{\mathrm{P}}=0.044\left(K_{\mathrm{I}} / \tau_{0}\right)^{2}$.

The contours of constant resolved shear stress on systems $\{110\}\langle 111\rangle$ and $\{112\}\langle 111\rangle$, which can become activated at higher temperature, shows that the largest zones over which the resolved shear stress on either of these two systems is larger than $\tau_{0}$ is of the same order as $R_{\mathrm{p}}$. The largest of these two zones are associated with the

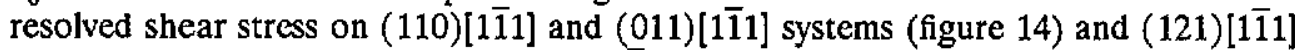
systems (figure 15) whose traces in the (101) plane form an angle of $35.26^{\circ}$ with the crack face. Although the critical resolved shear stress on either of these two systems is larger than that of the $\{110\}\langle 100\rangle$ system, the resolved shear stress on all members of these two set of systems is very large at the crack tip due to the stress singularity, thus one or the other of these two systems could become activated at sufficiently high temperature, relieving the stress singularity. Presumably, at lower temperatures, the maximum tensile stress $\sigma_{22}$ ahead of the tip becomes sufficiently large as to cause cleavage before such additional systems can be activated.

\section{Acknowledgment}

This study was supported by the Office of Naval Research, Mechanics Program, under grant number N00014-90-J-1379 to Harvard. The computational aspect of this work was partly performed on a Cray YMP computer at the Pittsburgh Supercomputing Center under a grant from NSF. The ABAQUS program was provided under an academic license by Hibbitt, Karlsson and Sorensen, Inc., Providence, Rhode Island.

\section{Appendix}

The field equations for the 5-sector asymptotic solution are as follows:

Elastic sector [1], $0 \leqslant \theta \leqslant \theta_{1}$ :

$$
\begin{aligned}
2 \mu u_{1}= & {\left[(1-\nu) Z_{1} \ln (r / X)-\nu Z_{2} \ln (r / Y)-(1-\nu) Z_{1}+\nu Z_{2}\right] r \cos \theta } \\
- & \frac{1}{2}\left[(3-2 \nu) Z_{1}+(1-2 \nu) Z_{2}\right] r \theta \sin \theta+W_{1} \\
2 \mu u_{2}=\left\{-\nu Z_{1}\right. & \left.\ln (r / X)+(1-\nu) Z_{2} \ln (r / Y)-\frac{1}{2}\left[(1-2 \nu) Z_{1}+(3-2 \nu) Z_{2}\right]\right\} r \sin \theta \\
+\frac{1}{2}\left[(1-2 \nu) Z_{1}+(3-2 \nu) Z_{2}\right] r \theta \cos \theta & \text { (A.1) }
\end{aligned}
$$




$$
\begin{aligned}
& \sigma_{11}=Z_{1} \ln (r / X)+\frac{1}{2}\left(Z_{1}+Z_{2}\right) \sin ^{2} \theta \\
& \sigma_{22}=Z_{2} \ln (r / Y)-\frac{1}{2}\left(Z_{1}+Z_{2}\right) \sin ^{2} \theta \\
& \sigma_{12}=-\frac{1}{2}\left(Z_{1}-Z_{2}\right) \theta-\frac{1}{4}\left(Z_{1}+Z_{2}\right) \sin 2 \theta
\end{aligned}
$$

Plastic sector [2], $\theta_{1} \leqslant \theta \leqslant \theta_{2}$ :

$$
\begin{aligned}
& \begin{aligned}
2 \mu u_{1}= & \left((1-\nu) Z_{1} \ln \left|\frac{r \sin \theta}{X \sin \theta_{1}}\right|-\nu Z_{2} \ln \left|\frac{r \cos \theta}{Y \cos \theta_{1}}\right|\right. \\
& \left.+\frac{1}{2}\left(Z_{1}+Z_{2}\right) \sin ^{2} \theta_{1}+\nu Z_{2}\right) r \cos \theta
\end{aligned} \\
& \quad+\left[\tau_{0}-(1-\nu) Z_{1} \cot \theta_{1}-(1-\nu)\left(Z_{1}+Z_{2}\right) \theta_{1}\right] r \sin \theta+W_{1} \\
& 2 \mu u_{2}=\left(-\nu Z_{1} \ln \left|\frac{r \sin \theta}{X \sin \theta_{1}}\right|+(1-\nu) Z_{2} \ln \left|\frac{r \cos \theta}{Y \cos \theta_{1}}\right|\right. \\
& \left.\quad-\frac{1}{2}\left(Z_{1}+Z_{2}\right) \sin ^{2} \theta_{1}+\nu Z_{1}\right) r \sin \theta \\
& \quad+\left[\tau_{0}-(1-\nu) Z_{2} \tan \theta_{1}+(1-\nu)\left(Z_{1}+Z_{2}\right) \theta_{1}\right] r \cos \theta \\
& \sigma_{11}=Z_{1} \ln \left|\frac{r \sin \theta}{X \sin \theta_{1}}\right|+\frac{1}{2}\left(Z_{1}+Z_{2}\right) \sin ^{2} \theta_{1} \\
& \sigma_{22}=Z_{2}\left|\frac{r \cos \theta}{Y \cos \theta_{1}}\right|-\frac{1}{2}\left(Z_{1}+Z_{2}\right) \sin ^{2} \theta_{1} \\
& \sigma_{12}=+\tau_{0} \\
& 4 \mu \epsilon_{12}^{\mathrm{p}}=(1-\nu)\left[Z_{1}\left(\cot \theta-\cot \theta_{1}\right)+Z_{2}\left(\tan \theta-\tan \theta_{1}\right)\right] .
\end{aligned}
$$

Elastic sector [3], $\theta_{2} \leqslant \theta \leqslant 90.0^{\circ}$ :

$$
\begin{gathered}
\begin{aligned}
2 \mu u_{1}=( & (1-\nu) Z_{1} \ln \left|\frac{r \sin \theta_{2}}{X \sin \theta_{1}}\right|-\nu Z_{2} \ln \left|\frac{r \cos \theta_{2}}{Y \cos \theta_{1}}\right| \\
& \left.-(1-\nu) Z_{1}+\nu Z_{2}+\frac{\left(Z_{1}+Z_{2}\right)}{2}\left(\sin ^{2} \theta_{1}-\sin ^{2} \theta_{2}\right)\right) r \cos \theta \\
& -\left\{\tau_{0}+(1-\nu) Z_{1}\left(\cot \theta_{1}-\cot \theta_{2}\right)-\left(Z_{1}-Z_{2}\right) \pi / 4\right. \\
& \left.+(1-\nu)\left(Z_{1}+Z_{2}\right)\left(\theta_{1}-\theta_{2}\right)+\frac{1}{2}\left[(3-2 \nu) Z_{1}+(1-2 \nu) Z_{2}\right] \theta\right\} r \sin \theta+W_{1}
\end{aligned} \\
\begin{aligned}
2 \mu u_{2}=(-\nu & Z_{1} \ln \left|\frac{r \sin \theta_{2}}{X \sin \theta_{1}}\right|+(1-\nu) Z_{2} \ln \left|\frac{r \cos \theta_{2}}{Y \cos \theta_{1}}\right| \\
& \left.-\frac{1}{2}\left[(1-2 \nu) Z_{1}+(3-2 \nu) Z_{2}\right]+\frac{1}{2}\left(Z_{1}+Z_{2}\right)\left(\sin ^{2} \theta_{2}-\sin ^{2} \theta_{1}\right)\right) r \sin \theta \\
& \quad-\left(\tau_{0}+(1-\nu) Z_{1}\left(\cot \theta_{2}-\cot \theta_{1}\right)-\left(Z_{1}-Z_{2}\right) \pi / 4\right. \\
& \left.\left.+(1-\nu)\left(Z_{1}+Z_{2}\right)\left(\theta_{2}-\theta_{1}\right)-\frac{1}{2}\left[(1-2 \nu) Z_{1}+(3-2 \nu) Z_{2}\right) \theta\right]\right\} r \cos \theta
\end{aligned} \\
\sigma_{11}=Z_{1} \ln \left|\frac{r \sin \theta_{2}}{X \sin \theta_{1}}\right|+\frac{1}{2}\left(Z_{1}+Z_{2}\right)\left(\sin ^{2} \theta-\sin ^{2} \theta_{2}+\sin ^{2} \theta_{1}\right) \\
\sigma_{22}=Z_{2}\left|\frac{r \cos \theta_{2}}{Y \cos \theta_{1}}\right|-\frac{1}{2}\left(Z_{1}+Z_{2}\right)\left(\sin ^{2} \theta-\sin ^{2} \theta_{2}+\sin ^{2} \theta_{1}\right) \\
\sigma_{12}=-\tau_{0}+\frac{1}{2}\left(Z_{1}-Z_{2}\right)(\pi / 2-\theta)-\frac{1}{4}\left(Z_{1}+Z_{2}\right) \sin 2 \theta
\end{gathered}
$$


Plastic sector [4], $90.0^{\circ} \leqslant \theta \leqslant \theta_{4}$

$$
\begin{aligned}
& 2 \mu u_{1}=(1-\nu) Z_{1} r \cos \theta \ln \left|\frac{r \sin \theta \sin \theta_{2}}{X \sin \theta_{1}}\right| \\
& +\left[\frac{1}{2}(1-\nu)\left(Z_{1}+Z_{2}\right)\left(\cos ^{2} \theta_{2}+\sin ^{2} \theta_{1}\right)+\frac{1}{2} \nu Z_{1} \sin ^{2} \theta_{4}\right] r \cos \theta \\
& -\left[\tau_{0}+(1-\nu) Z_{1}\left(\cot \theta_{1}-\cot \theta_{2}\right)+(1-\nu)\left(Z_{1}+Z_{2}\right)\right. \\
& \left.\times\left(\pi / 2-\theta_{2}+\theta_{1}\right)\right] r \sin \theta+W_{1} \\
& 2 \mu u_{2}=-\nu Z_{1} r \sin \theta \ln \left|\frac{r \sin \theta \sin \theta_{2}}{X \sin \theta_{1}}\right| \\
& -\left[(\nu / 2)\left(Z_{1}+Z_{2}\right)\left(\cos ^{2} \theta_{2}+\sin ^{2} \theta_{1}\right)+\frac{1}{2}(1-\nu) Z_{1} \sin ^{2} \theta_{4}-\nu Z_{1}\right] r \sin \theta \\
& -\left[\tau_{0}+(1-\nu) Z_{1}\left(\cot \theta_{4}+\cot \theta_{2}-\cot \theta_{1}\right)-(1-\nu)\left(Z_{1}+Z_{2}\right)\right. \\
& \left.\times\left(\pi / 2-\theta_{2}+\theta_{1}\right)\right] r \cos \theta+W_{2} \\
& \sigma_{11}=Z_{1} \ln \left|\frac{r \sin \theta \sin \theta_{2}}{X \sin \theta_{1}}\right|+\frac{1}{2}\left(Z_{1}+Z_{2}\right)\left(\sin ^{2} \theta_{1}+\cos ^{2} \theta_{2}\right) \\
& \sigma_{22}=\left(-Z_{1} / 2\right) \sin ^{2} \theta_{4} \\
& \sigma_{12}=-\tau_{0} \\
& 4 \mu \epsilon_{12}^{\mathrm{p}}=(1-\nu) Z_{1}\left(\cot \theta-\cot \theta_{4}\right) \text {. }
\end{aligned}
$$

Elastic sector $[5], \theta_{4} \leqslant \theta \leqslant 180.0^{\circ}$ :

$$
\begin{aligned}
2 \mu u_{1}=(1-\nu) & Z_{1} r \cos \theta \ln \left|\frac{r \sin \theta_{4} \sin \theta_{2}}{X \sin \theta_{1}}\right| \\
& -(1-\nu)\left[Z_{1}+\left(Z_{1} / 2\right) \sin ^{2} \theta_{4}-\frac{1}{2}\left(Z_{1}+Z_{2}\right)\left(\cos ^{2} \theta_{2}+\sin ^{2} \theta_{1}\right)\right] r \cos \theta \\
& +\left\{(1-\nu) Z_{1}\left(\cot \theta_{4}+\cot \theta_{2}-\cot \theta_{1}\right)+\left[\nu Z_{1}-(1-\nu) Z_{2}\right] \pi / 2\right. \\
& \left.+(1-\nu) Z_{1} \theta_{4}+(1-\nu)\left(Z_{1}+Z_{2}\right)\left(\theta_{2}-\theta_{1}\right)-\frac{1}{2}(3-2 \nu) Z_{1} \theta\right\} r \sin 0+W_{1}
\end{aligned}
$$$$
2 \mu u_{2}=-\nu Z_{1} r \sin \theta \ln \left|\frac{r \sin \theta_{4} \sin \theta_{2}}{X \sin \theta_{1}}\right|-\left[\frac{1}{2}(1-2 \nu) Z_{1}-(\nu / 2) Z_{1} \sin ^{2} \theta_{4}\right.
$$$$
\left.+(\nu / 2)\left(Z_{1}+Z_{2}\right)\left(\cos ^{2} \theta_{2}+\sin ^{2} \theta_{1}\right)\right] r \sin \theta-\left\{(1-\nu) Z_{1}\right.
$$$$
\times\left(\cot \theta_{4}+\cot \theta_{2}-\cot \theta_{1}\right)-\left[(2-\nu) Z_{1}+(1-\nu) Z_{2}\right] \pi / 2
$$$$
\left.+(1-\nu) Z_{1} \theta_{4}+(1-\nu)\left(Z_{1}+Z_{2}\right)\left(\theta_{2}-\theta_{1}\right)-\frac{1}{2}(1-2 \nu) Z_{1} \theta\right\} r \cos \theta+W_{2}
$$

$\sigma_{11}=Z_{1} \ln \left|\frac{r \sin \theta_{4} \sin \theta_{2}}{X \sin \theta_{1}}\right|+\left(Z_{1} / 2\right)\left(\sin ^{2} \theta-\sin ^{2} \theta_{4}\right)+\frac{1}{2}\left(Z_{1}+Z_{2}\right)\left(\cos ^{2} \theta_{2}+\sin ^{2} \theta_{1}\right)$

$\sigma_{22}=-\left(Z_{1} / 2\right) \sin ^{2} \theta$

$\sigma_{12}=\left(Z_{1} / 2\right)(\pi-\theta)-\left(Z_{1} / 4\right) \sin 2 \theta$.

In the above equations all the constants have been written in terms of $Z_{1}$ and $Z_{2}$ of the plastic sector [2]. The constants $X$ and $Y$ are used to denote the constants which are left undetermined by the asymptotic analysis, namcly $3 B_{1}-D_{1}=-Z_{1} \ln X$ and $B_{1}+D_{1}=-Z_{2} \ln Y$, where the constant $B_{1}$ and $D_{1}$ refer to the values of these constants in the elastic sector [1]. 


\section{References}

ABAQUS Manual version 4.8, 1989 (Providence, RI: Hibbitt, Karlsson and Sorensen, Inc.)

Fleisher R L, Dimiduk D M and Lipsitt H A 1989 Intermetallic compounds for strong high-temperature materials: status and potential Ann. Rev. Mater. Sci. 19 231-63

Fu C L and Yoo M H 1991 First-principles investigation of mechanical behavior of B2 type aluminides: FeAl and NiAl Material Research Society Symp. Proc. (Baston, MA, 27-30 November 1990) vol 213 High-Temperature Ordered Intermetallic Alloys $N$ (Pittsburg, PA: Materials Research Society) pp 667-72

George E P and Liu C T 1990 Brittle fracture and grain boundary chemistry of microalloyed NiA $J$. Mater. Res. 5 754-62

Guha S, Munroe P R and Baker I 1989 Improving the low temperature ductility of NiAl Material Research Society Symp. Proc. (Boston, MA, 29 November-1 December 1988) vol 133 High-Temperature Ordered Intennetallic Alloys III (Pittsburg, PA: Materials Research Society) pp 633-8

Huang Y 1991 A user-material subroutine incorporating single crystal plasticity in the ABAQUS finite element program, $M E C H-178$, (Harvard University: Division of Applied Sciences)

Kanchi M B, Zienkiewicz O C and Owen D R J 1978 The visco-plastic approach to problems of piasticity and creep involving geometric non-linear effects Int. J. Numerical Methods Eng. 12 169-81

Miracle D B, Russell S and Law C C 1989 Slip system modification in NiAl Material Research Society Symp. Proc. (Boston, MA, 29 November-1 December 1988) vol 133 High-Temperature Ordered Intermetallic Alloys III (Pittsburg, PA: Materials Research Society) pp 225-30

Noebe R D, Cullers C L and Bowman R R 1992 The effect of strain rate and temperature on the tensile properties of NiAl $J$. Mater. Res. 7 605-12

Peirce D, Asaro R J and Needleman A 1983 Material rate dependence and localized deformation in crystalline solids Acta Metall. 31 1951-76

Rice J R 1987 Tensile crack tip fields in elastic-ideally plastic crystals Mech. Mater. 6 317-35

Russell S M, Law C C and Blackburn M J 1989 The effect of cobalt on martensitic toughening parameters in NiAl Material Research Society Symp. Proc. (Bostom, MA, 29 November-1 December 1988) vol 133 High-Temperature Ordered Intermetallic Alloys III (Pittsburg, PA: Materials Research Society) pp $627-32$

Vedula K, Hahn K H and Boulogne B 1989 Room temperature tensile ductility in polycrystalline B2 NiAl Material Research Society Symp. Proc. (Boston, MA, 29 November-1 December 1988) vol 133 HighTemperature Ordered Internnetallic Alloys III (Pittsburg, PA: Materials Research Society) pp 299-304

Vedula K and Stephens J R 1987 B2 aluminides for high temperature applications Material Research Society Symp. Proc. (Boston, MA, 2-4 December 1986) vol 81 High-Temperature Ordered Intennetallic Alloys II (Pittsburg, PA: Materials Research Society) pp 381-91 